Article

\title{
Breast Milk Supply of MicroRNA Associated with Leptin and Adiponectin Is Affected by Maternal Overweight/Obesity and Influences Infancy BMI
}

\author{
Rocío Zamanillo ${ }^{1,2}$, Juana Sánchez ${ }^{1,3,4}$, Francisca Serra ${ }^{1,3,4, * \mathbb{C}}$ and Andreu Palou 1,3,4 (D) \\ 1 Laboratory of Molecular Biology, Nutrition and Biotechnology (Group of Nutrigenomics and Obesity), \\ University of the Balearic Islands, 07122 Palma, Spain; oicorzc@gmail.com (R.Z.); joana.sanchez@uib.es (J.S.); \\ andreu.palou@uib.es (A.P.) \\ 2 Department of Health, Valencian International University (VIU), 46002 Valencia, Spain \\ 3 CIBER de Fisiopatología de la Obesidad y Nutrición (CIBERobn), Instituto de Salud Carlos III (ISCIII), \\ 28029 Madrid, Spain \\ 4 Instituto de Investigación Sanitaria Illes Balears (IdISBa), 07120 Palma, Spain \\ * Correspondence: francisca.serra@uib.es; Tel.: +34 971173051
}

Received: 30 July 2019; Accepted: 7 October 2019; Published: 28 October 2019

\begin{abstract}
Breast milk constitutes a dietary source of leptin, adiponectin and microRNAs (miRNAs) for newborns. Expression of miRNAs previously associated with maternal obesity, leptin or adiponectin function were assessed and their impact on infant weight analyzed. Milk samples were collected (at month 1, 2, and 3) from a cohort of 59 healthy lactating mothers (38 normal-weight and 21 overweight/obese (BMI $\geq 25)$ ), and infant growth was followed up to 2 years of age. Thirteen miRNAs, leptin and adiponectin were determined in milk. Leptin, adiponectin and miRNA showed a decrease over time of lactation in normal-weight mothers that was altered in overweight/obesity. Furthermore, negative correlations were observed in normal-weight mothers between the expression of miRNAs in milk and the concentration of leptin or adiponectin, but were absent in overweight/obesity. Moreover, miRNAs negatively correlated with infant BMI only in normal-weight mothers (miR-103, miR-17, miR-181a, miR-222, miR-let7c and miR-146b). Interestingly, target genes of milk miRNAs differently regulated in overweight/obesity could be related to neurodevelopmental processes. In conclusion, a set of miRNAs present in breast milk, in close conjunction with leptin and adiponectin, are natural bioactive compounds with the potential to modulate infant growth and brain development, an interplay that is disturbed in the case of maternal overweight/obesity.
\end{abstract}

Keywords: breast milk microRNA; maternal obesity; leptin; adiponectin; infant growth

\section{Introduction}

Human milk supplies a number of nutrients and bioactive compounds that drive the development of infants by modulating their metabolic programming and handling genetic, epigenetic and environmental cues, and therefore preparing them for the best outcome in adulthood. Maternal overweight or obesity is deemed to influence the developmental program which could lead to unsuccessful proper growth with consequences in adult life and performance [1].

MicroRNAs (miRNAs) are short (21-25 nucleotides) non-coding RNA with the potential to modulate transcription and translation of target miRNA. The recent discovery of miRNAs in breast milk and their high stability has driven attention to their physiological function in lactation [2]. Breast milk constitutes a rich dietary source of miRNAs delivered to new born mammals [3,4]. Although still controversial, milk miRNAs may have the potential of being absorbed by the immature intestinal tract where they may exert their action at a systemic level during early postnatal life [5-8]. miRNA's plasma 
profile has been shown to be altered in gestational [9] and childhood obesity [10,11]. In addition, miRNA's profile in breast milk may be modulated by both maternal body weight [12] and diet $[8,13]$. However, little is known regarding the miRNA's profile in human breast milk and the potential impact on obesity in infants.

Leptin and adiponectin are two hormones present in maternal milk with a key role in the regulation of energy balance. Leptin has been the focus of intensive research in order to characterize its protective anti-obesity role, particularly during developmental stages $[14,15]$. Breast milk leptin programs the offspring to be more protected against obesity and other metabolic alterations later on life [16-19]. Here, we hypothesize that breast milk miRNAs, mostly the ones associated with leptin and adiponectin function, could be altered in overweight/obese lactating mothers and thereby affect growth performance in their infants. Results showed that maternal obesity disturbed the breast milk supply of these miRNAs and their association with milk levels of leptin and adiponectin, as well as their impact on infant BMI at two years of age.

\section{Materials and Methods}

\subsection{Participants and Milk Sample Collection}

Fresh milk was obtained from 59 healthy lactating mothers on day 30, 60 and 90 of lactation or as close as possible. Milk was collected (3-12 mL) into sterile recipients between 9 a.m. and 2 p.m. and was kept at $4{ }^{\circ} \mathrm{C}$, transported to the lab the same day and then frozen in working aliquots at $-80^{\circ} \mathrm{C}$ until further analysis. Milk collection was obtained during the scheduled visits of participants to the midwives. There the researcher involved ( $R$ Zamanillo) was present and was responsible for sampling procedure and preservation. To cause minimal disturbances to both mother and the breastfed infant, no specific conditions were imposed on the volunteers for milk sampling. Therefore, milk was collected at the best time of the morning for the mother, normally from both breasts and, usually while the baby was breastfeeding from one side, a milk cup (Medela, Barcelona, Spain) collected the milk from the other breast. In this way, milk collection was performed in a natural and physiological manner and did not cause maternal stress. In addition, a manual breast pump (Medela, Barcelona, Spain) was available during the visit to mothers who preferred this system. In these cases, mothers pumped their breasts after feeding the baby, because it was easier to drain the milk.

Mother-children pairs were enrolled for participation during planned postnatal controls with their midwives. All participants gave written consent. and the protocol was approved by the Ethics Committee of Clinical Research of Balearic Islands (CEIC) (agreement IB 1645/11 PI, July 2011).

Anthropometric data from mothers and infants were recorded at birth and at the time of sample collection. Follow-up contact was scheduled and performed by phone or e-mail in order to track growth performance of the infants up to 2 years of age. Maternal BMI was calculated as the mean of BMI (body weight self-reported) prior to pregnancy and at months 1,2 and 3 of lactation. This average BMI was considered to be a good indicator of maternal obesity classification since it reflects perinatal maternal body mass index.

\section{2. miRNA Analysis}

Firstly, $100 \mu \mathrm{L}$ of breast milk samples were allowed to thaw in iced water and were homogenized by vortexing for $10 \mathrm{~s}$. Then, small RNAs from breast milk were purified with the microRNA isolation kit mirVana (Kit AM1561, Ambion) following the manufacturer's instructions and a previously described procedure [2]. The final filtered sample containing small RNAs was eluted in nuclease-free water preheated at $95^{\circ} \mathrm{C}$. The integrity and concentration of RNA was assessed by spectrophotometry using Nanodrop (NanoDrop Technologies, Wilmington, DE, USA) and by capillary electrophoresis using the Small RNA kit (Chip kit 5067-1548) for the Agilent 2100 Bioanalyzer. Quality of RNA preparations was assessed by observing electropherogram profiles; samples showing irregular ones were discarded. 
Reverse transcription (RT) and quantitative PCR (qPCR) of selected miRNAs were performed according to the manufacturer's protocol. In brief, samples were adjusted to $2.5 \mathrm{ng} / \mu \mathrm{L}$ of small RNAs. Then, $5 \mu \mathrm{L}$ was reverse-transcribed to cDNA in a $4 \mu \mathrm{L}$ reaction mix (TaqMan microRNA Reverse Transcription kit, Applied Biosystems) together with $1.5 \mu \mathrm{L}$ of the miRNA-specific RT primers provided with the TaqMan MicroRNA Assay (Applied Biosystems). RT reactions were performed using an Applied Biosystems 2720 Thermal Cycler (Applied Biosystems) under the following conditions: $16{ }^{\circ} \mathrm{C}$ for $30 \mathrm{~min} ; 42^{\circ} \mathrm{C}$ for $30 \mathrm{~min}$ and $85^{\circ} \mathrm{C}$ for $5 \mathrm{~min}$. Then, $2 \mu \mathrm{L}$ of the miRNA-specific cDNA from RT reaction was amplified with the TaqMan Universal PCR master mix and the respective specific probe provided in the TaqMan MicroRNA Assay (Applied Biosystems). Amplification was initiated at $95^{\circ} \mathrm{C}$ for $10 \mathrm{~min}$ followed by 40 cycles consisting of denaturation at $95^{\circ} \mathrm{C}$ for $15 \mathrm{~s}$, followed by annealing and extension at $60^{\circ} \mathrm{C}$ for $1 \mathrm{~min}$. PCR was performed in a StepOnePlusTM Real-Time PCR System (Applied Biosystems). The mature sequence and manufacturer's reference assay of the selected miRNA are shown in Table S1.

Selection of miRNAs was based on the literature and the following criteria: they are potential or demonstrated targets for mRNA of leptin (LEP), adiponectin (ADIPOQ), and their respective receptors (LEPR, ADIPOR1 and ADIPOR2); and have been found in fluids (plasma or milk) or associated with obesity $[2,4,20]$. U6 snRNA was selected as an endogenous control in the comparative cycle threshold method [13]. Expression of hsa-miR-539-5p is tissue specific [21] and was included as a negative control. To ensure reproducibility and to check for day-to-day and plate-to-plate variability, a set of samples were repeated in each RT (two per plate) as internal controls. A maximum of $10 \%$ variability between plates was considered acceptable and, on average, the ratio between plates of those samples was $1.001 \pm 0.007(n=18)$.

In silico target prediction analysis for miRNAs of interest was carried out using miRror 2.0 Suite [22]. Then, a functional enrichment analysis of these targets was conducted by Gene Ontology (GO) annotation and GlueGo+CluePedia Cytoscape plugin [23-25].

\subsection{Analytical Methods}

Milk triglycerides (TG) were determined by commercially available kits (K622-100 from BIOVISION (Milpitas, California)). Individual fatty acids were determined by gas chromatography (GC) in duplicate following the previously described method [26].

Leptin and adiponectin concentration in whole milk samples was determined using commercially available kits. Human Leptin Quantikine ELISA Kit (Quantikine, DLP00) (R\&D Systems Europe, Ltd., Abingdon, UK) and ADIPOQ (Human) High Sensitivity ELISA Kit (Abnova, KA0017) (Abnova, Taipei City, Taiwan) were used following the manufacturers' indications and previously validated protocols in our laboratory $[16,27]$.

\subsection{Statistical Analysis}

Normal distribution of data was tested by Kolmogorov-Smirnov (when $n \geq 40$ ) or Shapiro-Wilk (when $n<40$ ) tests. When data did not follow a normal distribution, variables were log- or lntransformed and then considered appropriate. Homogeneity of variances was estimated by Levene's test. Repeated measures analysis of variance (rANOVA) was used to assess the impact of time ( $\mathrm{T}$, month of lactation) and maternal body weight (W, using BMI as a surrogate) on expression of miRNA and analytical variables, and was followed by pairwise comparison to detect differences between months. The associations between miRNA expression and perinatal and maternal variables were tested using Spearman's correlation test. All statistical analyses were performed using IBM SPSS Statistics 20 and a $p$-value $<0.05$ was considered statistically significant. 


\section{Results}

\subsection{Participant Characteristics}

Fifty-nine mothers and their infants completed the study, 64\% of whom were normal-weight $(\mathrm{BMI}<25)$ and $36 \%$ overweight or obese (BMI $\geq 25)$. Most of them were of Caucasian origin $(90 \%)$, had a natural birth (83\%), and at term (86\%). Almost equal distribution of sexes between infants $(53 \%$ girls) was observed. At the time of the first milk sampling ( 1 month post-partum), most of the mothers were following exclusive breastfeeding (86\%), which showed a decreasing trend during the subsequent sampling points (85\% and $78 \%$ at months 2 and 3, respectively). Table S2 shows the characteristics of the whole population, segregated by BMI.

When considering groups by maternal BMI, no major differences were found regarding maternal and gestational age. A slight tendency towards a higher BMI was found in children from overweight/obese mothers, but this did not attain any statistical significance throughout the period studied (Table S3).

\subsection{Expression Levels of miRNAs and Leptin and Adiponectin Concentrations in Breast Milk}

Thirteen miRNAs, of the twenty-six chosen for this study, were detected in breast milk showing a wide range of activity. miR-30a, miR-146b, miR-let7b, and miR-148a were among the most abundant, whereas miR-27a and miR-27b presented the lowest expression, being around two orders of magnitude lower. Analysis of the whole population showed a decrease in the expression of six miRNAs (miR-222, miR-103, miR-200b, miR-17, miR-let7c, and miR-146b) associated with time of lactation $(p<0.05)$ (Table 1). When maternal BMI was taken into account, a pattern of decreased expression over time, mainly associated with overweight/obesity, was more apparent. Post-hoc analysis of lactating mothers grouped by BMI showed an increase in miR-30a expression in normal-weight mothers $(p<0.05)$ over time that was disturbed in overweight/obese; and a decreased expression of miR-146b that was significant in both groups $(p<0.05)$. The changes observed in the rest of miRNAs showed a general tendency to decrease throughout lactation in normal-weight conditions, but the presence of overweight/obesity unsettled the expression profile. Thus, a smooth decline over time that did not attain statistical significance in normal-weight mothers was observed for the miRNAs analyzed. In contrast, in overweight/obese mothers, expression at month 1 of lactation was much higher but had declined by month 2 . This pattern was statistically significant in the overweight/obese population for miR-222, miR-103, miR-17, miR-let7a, and miR-let7c $(p<0.05)$ (Table 1). An overview of the relative percentage of each miRNA the three months studied, shown as 100\% stacked bars for each miRNA, allows an easier comparison of miRNA value between normal weight and overweight/obese women (Figure 1).

Milk leptin concentration in the whole population showed a decrease over time of lactation $(p<0.05)$ which was confirmed in normal-weight $(p<0.05)$ but was absent in overweight/obese mothers. Furthermore, overweight/obese mothers showed higher levels of leptin throughout lactation (2.8 higher at month 1) $(p<0.05)$ than normal-weight mothers. Adiponectin concentration was not different between normal-weight and overweight/obese mothers at month 1, but normal-weight mothers showed a decrease over time $(\approx 20 \%, p<0.05)$ which was not seen in overweight/obese (Table 2). 


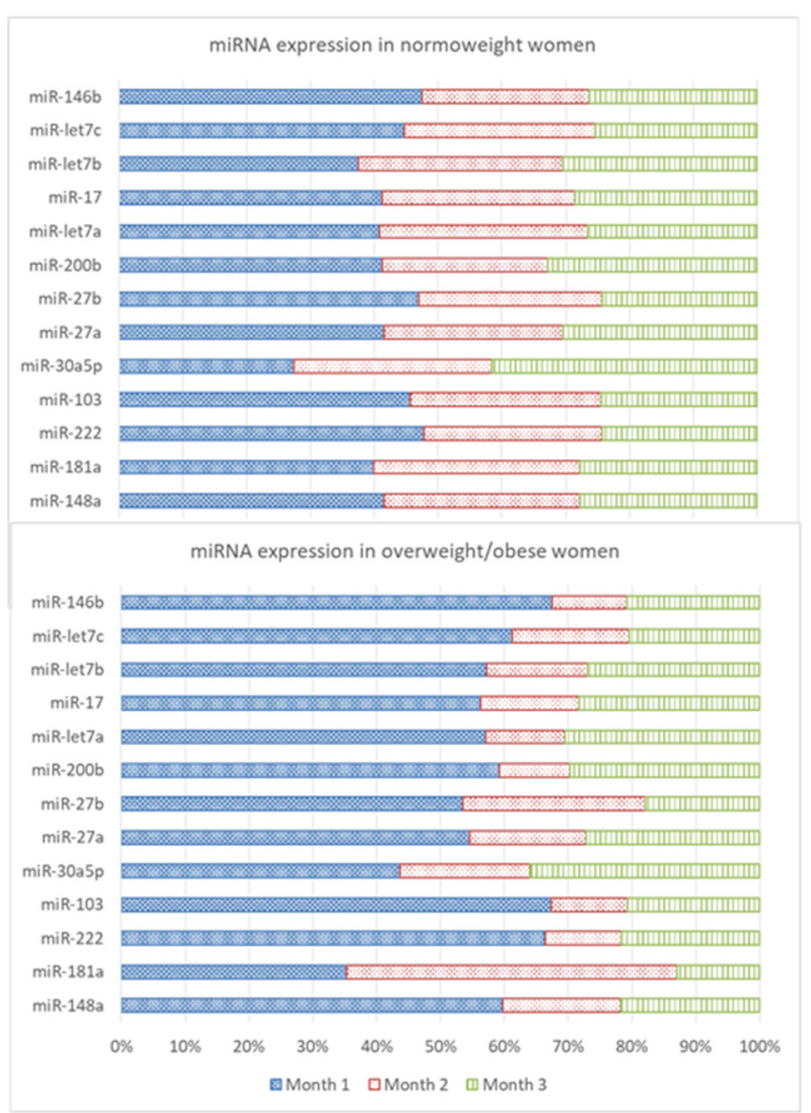

Figure 1. Stacked chart showing the relative percentage of expression of miRNA at month 1, 2, and 3 in normal weight and overweight/obese mothers. The cumulative value of each stacked bar has been defined as $100 \%$.

\subsection{In Silico Analysis of Potential miRNA Target Genes}

To further investigate the potential roles of the miRNAs affected by maternal BMI and those that displayed an altered milk level profile over time due to maternal overweight/obesity (miR-let7a, miR-103, miR-222, miR-17, miR-146b, miR-30a), we checked their predicted target genes using miRror 2.0 Suite.

According to miRror, 487 genes are targeted by at least 1 of these miRNAs. All these potential target genes were tested for GO pathway analysis using GlueGo + CluePedia Cytoscape plugin. The genes predicted to be targets of the miRNAs were significantly enriched for 13 term groups that constituted 9 functionally grouped networks: epithelial cell development, neuromuscular process, Wnt signaling pathway and calcium modulating pathway, regulation of macroautophagy, central nervous system neuron differentiation, lipid particle organization, Golgi vesicle transport, cranial nerve development, and cell morphogenesis involved in differentiation (Figure 2). 
A)

\begin{tabular}{|c|c|c|c|c|}
\hline GOT erm & $\begin{array}{l}\text { Term } \\
\text { PValue }\end{array}$ & $\begin{array}{c}\text { Term PValue } \\
\text { Corrected }\end{array}$ & $\begin{array}{c}\text { Group } \\
\text { PValue }\end{array}$ & $\begin{array}{c}\text { Group PValue } \\
\text { Corrected }\end{array}$ \\
\hline $\begin{array}{l}\text { Epithelial cell development } \\
\text { Nearromuscular process }\end{array}$ & $\begin{array}{l}0.00000 \\
0.00002\end{array}$ & \begin{tabular}{l|}
0.00037 \\
0.00530
\end{tabular} & $\begin{array}{l}0.00000 \\
0.00002\end{array}$ & $\begin{array}{l}0.00001 \\
0.000012\end{array}$ \\
\hline $\begin{array}{l}\text { Wnt signaling pathway, calcium } \\
\text { modulating pathway }\end{array}$ & 0.00001 & 0.00387 & 0.00001 & 0.00012 \\
\hline Regulation of macroautophagy & 0.00014 & 0.03565 & 0.00014 & 0.00041 \\
\hline $\begin{array}{l}\text { Central nervous systen } \\
\text { differentiation }\end{array}$ & 0.00003 & 0.00918 & 0.00003 & 0.00014 \\
\hline $\begin{array}{l}\text { Lipid particle organization } \\
\text { Gold vesicle transport }\end{array}$ & 0.00018 & $\begin{array}{ll}0.04576 \\
0.00839\end{array}$ & 0.00018 & $\begin{array}{ll}0.00018 \\
0.00016\end{array}$ \\
\hline $\begin{array}{l}\text { Gologi vesicle transpoc } \\
\text { Nerve development }\end{array}$ & 0.00003 & 0.04466 & & \\
\hline Cranial nerve development & 0.00012 & $\begin{array}{l}0.03049 \\
0.04607\end{array}$ & \multirow{4}{*}{0.00002} & \multirow{4}{*}{0.00011} \\
\hline $\begin{array}{l}\text { Developmental growth } \\
\text { Developmental growth involved in }\end{array}$ & $\frac{0.00018}{0.00019}$ & 0.04607 & & \\
\hline $\begin{array}{l}\text { Cel morphogenesis involved in } \\
\text { differentiation }\end{array}$ & 0.00001 & 0.00357 & & \\
\hline Neuron projection morphogenesis & 0.00016 & 0.0426 & & \\
\hline
\end{tabular}

B)

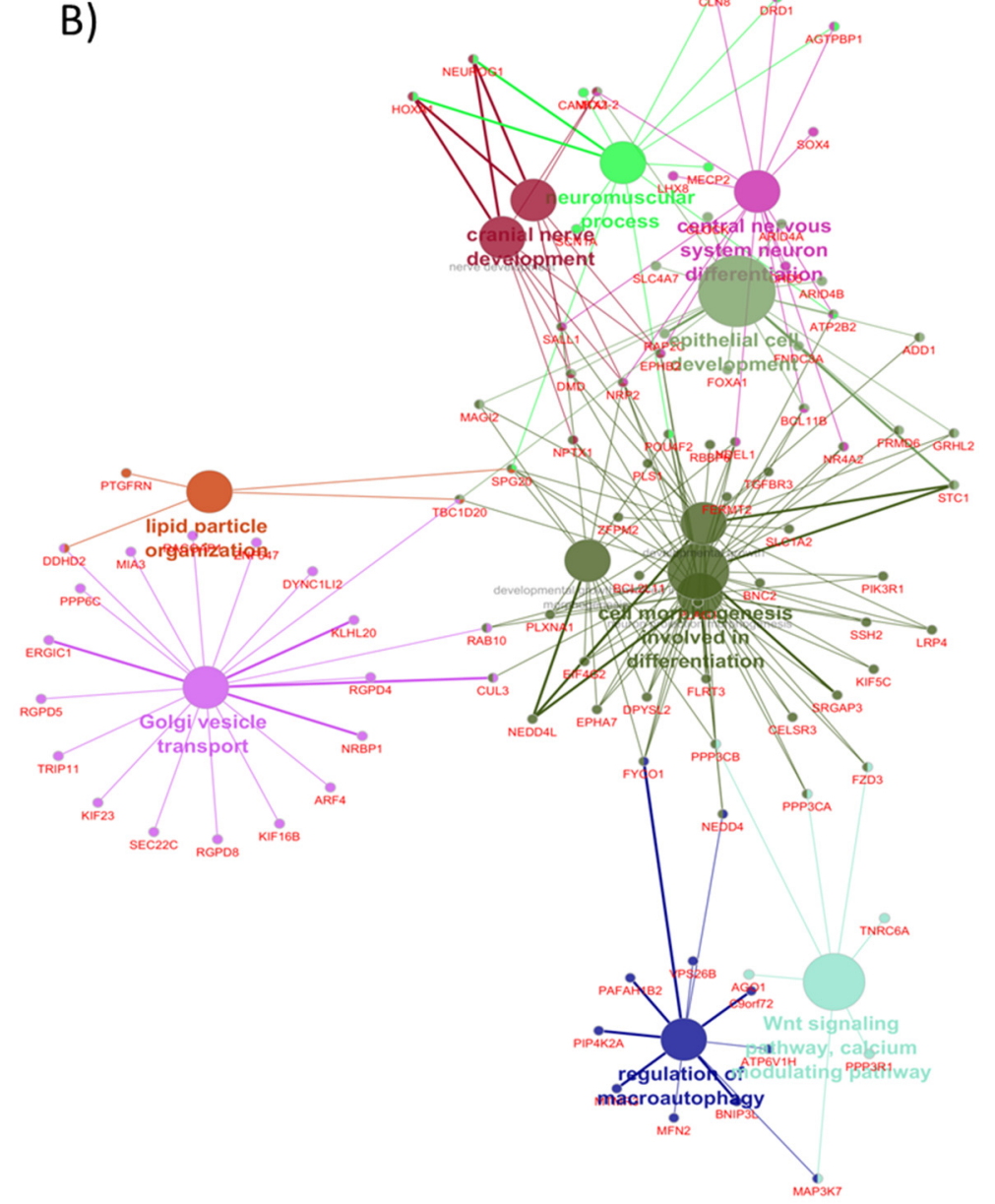

Figure 2. Functional enrichment analysis Gene Ontology (GO) terms for biological process of the predicted target genes of the six of miRNAs which showed altered profiles over time associated with maternal obesity. (A) Information on GO terms. $p$ values before and after Bonferroni step down correction are indicated. (B) Functionally grouped networks with terms as nodes; the most significant terms in the group are labelled in bold. Analysis was conducted using ClueGo + Clupedia app in Cytoscape [23-25] 


\subsection{Association Analysis of miRNAs with Key Parameters}

Correlation analysis was performed with breast milk fatty acid content to assess its potential relationship with miRNAs, as human milk miRNAs have been shown to be influenced by lipid content [5].

In normal-weight mothers, negative correlations between miRNAs expression and the monounsaturated fatty acid (MUFA) content in milk were observed at the first month of lactation, which attained statistical significance for miR-222, miR-103, miR-27b, miR-200b, miR-17, and miR-let7b $(p<0.05)$. At month 2 , positive correlations were found between milk saturated fatty acid content and miR-181a, miR-103, miR-let7a, miR-let7b, miR-let7c, and miR-17 $(p<0.05)$. Finally, at the third month of lactation, miRNAs expression was positively correlated with milk triglyceride concentration (miR-148a, miR-181a, miR-222, miR-103, miR-30a, miR-200b, miR-17, miR-let7b, miR-let7c, and miR-146b $(p<0.05)$ ). Interestingly, this pattern was totally distorted in overweight/obese mothers, in whom statistically significant correlations were only shown at month 2 of lactation; with MUFA positive for miR-103 and miR-200b $(p<0.05)$ and with polyunsaturated fatty acids (PUFA) negative for miR-let7a, miR-let7c, and miR-27a $(p<0.05)$. The whole set of data is included as Table S4.

Furthermore, the association of miRNAs, leptin, and adiponectin levels in milk we analyzed at 2 months of lactation and with infant growth (Table 3). Regarding the correlations of miRNAs and adipokines, results observed in the whole population were mirrored by the normal-weight population but were counteracted by overweight or obese status. Specifically, negative correlations were observed in normal-weight mothers between leptin and miRNAs levels in milk (miR-30a, miR27a, miR-27b, miR-181a, miR-222, miR-103, miR-17, and miR-146b); whereas none of them were found to be either statistically significant or negative in overweight/obese mothers (Table 3). Milk adiponectin negatively correlated with miR-148a, miR-let7a, miR-let7b, miR-103, and miR-17 $(p<0.05)$ in normal-weight mothers; however, no correlations were observed in overweight/obese ones. Interestingly, milk miRNAs were negatively correlated with infant BMI at 24 months of age in normal-weight mothers (miR-103, miR-17, miR-181a, miR-222, miR-let7c, and miR-146b $(p<0.05)$ ) whereas no correlations were found in overweight/obese. 
Table 1. MicroRNAs (miRNAs) in breast milk during lactation.

\begin{tabular}{|c|c|c|c|c|c|c|c|c|c|c|c|c|c|c|c|c|c|c|}
\hline & & \multicolumn{5}{|c|}{ Month 1} & \multicolumn{5}{|c|}{ Month 2} & \multicolumn{5}{|c|}{ Month 3} & \multicolumn{2}{|c|}{ Statistical Analysis } \\
\hline & & $\mathbf{N}$ & Min & $\operatorname{Max}$ & Mean & SD & $\mathbf{N}$ & Min & Max & Mean & SD & $\mathbf{N}$ & Min & Max & Mean & SD & Overall & $\begin{array}{c}\text { Normal/Obese } \\
\text { Weight }\end{array}$ \\
\hline \multirow[b]{2}{*}{ miR-148a } & Whole & 52 & 0.004 & 237.21 & 21.98 & 45.40 & 53 & 0.061 & 69.52 & 11.28 & 13.73 & 55 & 0.029 & 88.37 & 11.13 & 15.46 & & \\
\hline & Normal-weight & 34 & 0.004 & 124.38 & 15.77 & 24.35 & 36 & 0.063 & 69.52 & 11.69 & 14.62 & 36 & 0.262 & 62.59 & 10.54 & 12.13 & & \\
\hline \multirow{3}{*}{ miR-181a } & Whole & 53 & 0.024 & 34.72 & 2.93 & 5.36 & 54 & 0.060 & 53.90 & 3.33 & 10.11 & 55 & 0.041 & 14.48 & 1.49 & 2.22 & & \\
\hline & Normal-weight & 34 & 0.024 & 10.14 & 1.99 & 2.14 & 36 & 0.060 & 7.97 & 1.61 & 1.94 & 36 & 0.067 & 7.64 & 1.38 & 1.41 & & \\
\hline & Overweight/obese & 19 & 0.115 & 34.72 & 4.63 & 8.36 & 18 & 0.068 & 53.90 & 6.78 & 17.09 & 19 & 0.041 & 14.48 & 1.69 & 3.29 & & \\
\hline \multirow{2}{*}{ miR-222 } & Whole & 51 & 0.017 & 31.63 & $2.99^{\mathrm{a}}$ & 5.89 & 53 & 0.032 & 6.46 & $1.04^{\mathrm{a}, \mathrm{b}}$ & 1.14 & 54 & 0.024 & 9.75 & $1.20^{\mathrm{b}}$ & 1.63 & $\mathrm{~T}(p=0.020)$ & \\
\hline & Overweight/obese & 17 & 0.108 & 31.63 & $5.22^{\mathrm{a}}$ & 9.40 & 18 & 0.032 & 4.09 & $0.94^{\mathrm{ab}}$ & 1.05 & 18 & 0.024 & 9.75 & $1.70^{\mathrm{b}}$ & 2.60 & \multicolumn{2}{|c|}{$\mathrm{T}(p=0.006) \quad \mathrm{T}(p=0.029)$} \\
\hline \multirow{3}{*}{ miR-103 } & Whole & 52 & 0.019 & 21.09 & $2.21^{\mathrm{a}}$ & 3.81 & 54 & 0.036 & 7.05 & $0.90^{\mathrm{b}}$ & 1.25 & 54 & 0.013 & 7.70 & $0.91^{b}$ & 1.28 & $\mathrm{~T}(p=0.019)$ & \\
\hline & Normal-weight & 34 & 0.019 & 11.80 & 1.60 & 2.27 & 36 & 0.036 & 7.05 & 1.05 & 1.47 & 36 & 0.053 & 4.52 & 0.85 & 0.91 & \multirow{2}{*}{$\operatorname{TxW}(p=0.022)$} & \\
\hline & Overweight/obese & 18 & 0.105 & 21.09 & $3.36^{\mathrm{a}}$ & 5.61 & 18 & 0.070 & 2.02 & $0.59^{\mathrm{b}}$ & 0.52 & 18 & 0.013 & 7.70 & $1.03^{\mathrm{b}}$ & 1.83 & & $\mathrm{~T}(p=0.017)$ \\
\hline \multirow{3}{*}{ miR-30a } & Whole & 51 & 0.474 & 410.65 & 47.84 & 71.53 & 53 & 0.355 & 220.17 & 37.26 & 41.28 & 55 & 1.66 & 510.41 & 54.88 & 78.87 & & \\
\hline & Normal-weight & 33 & 0.474 & 112.54 & $34.48^{\mathrm{a}}$ & 34.18 & 35 & 1.64 & 220.17 & $39.04^{b}$ & 44.04 & 36 & 2.85 & 252.94 & $52.31^{\mathrm{b}}$ & 51.76 & \multirow{2}{*}{$\mathrm{TxW}(p=0.023)$} & $\mathrm{T}(p=0.008)$ \\
\hline & Overweight/obese & 18 & 1.70 & 410.65 & 72.33 & 108.94 & 18 & 0.355 & 128.04 & 33.81 & 36.27 & 19 & 1.66 & 510.41 & 59.75 & 115.82 & & \\
\hline miR-27a & Whole & 52 & 0.004 & 4.68 & 0.40 & 0.81 & 54 & 0.008 & 1.84 & 0.18 & 0.27 & 55 & 0.008 & 4.09 & 0.23 & 0.56 & & \\
\hline \multirow{3}{*}{$\mathrm{miR}-27 \mathrm{~b}$} & Whole & 52 & 0.003 & 1.44 & 0.19 & 0.27 & 54 & 0.002 & 1.50 & 0.11 & 0.21 & 52 & 0.002 & 0.65 & 0.08 & 0.11 & & \\
\hline & Normal-weight & 34 & 0.003 & 0.89 & 0.15 & 0.18 & 36 & 0.002 & 0.57 & 0.09 & 0.11 & 35 & 0.003 & 0.40 & 0.08 & 0.08 & \multirow{2}{*}{$\mathrm{T}(p=0.022)$} & \\
\hline & Overweight/obese & 18 & 0.007 & 1.44 & 0.26 & 0.39 & 18 & 0.004 & 1.50 & 0.14 & 0.34 & 17 & 0.002 & 0.65 & 0.09 & 0.16 & & \\
\hline \multirow{3}{*}{$\mathrm{miR}-200 \mathrm{~b}$} & Whole & 52 & 0.041 & 78.38 & $9.06^{a}$ & 14.69 & 49 & 0.002 & 24.40 & $3.68^{\mathrm{b}}$ & 4.48 & 53 & 0.066 & 40.98 & $5.87^{\mathrm{ab}}$ & 7.71 & \multirow[t]{3}{*}{$\mathrm{T}(p=0.026)$} & \\
\hline & Normal-weig & 34 & 0.088 & 36.53 & 6.87 & 8.35 & 32 & 0.007 & 24.40 & 4.33 & 5.17 & 34 & 0.138 & 23.98 & 5.46 & 5.12 & & \\
\hline & Overweight/obese & 18 & 0.041 & 78.38 & 13.20 & 22.01 & 17 & 0.002 & 8.00 & 2.46 & 2.46 & 19 & 0.066 & 40.98 & 6.60 & 11.07 & & \\
\hline \multirow{3}{*}{ miR-let7a } & Whole & 51 & 0.063 & 56.35 & 5.44 & 10.03 & 48 & 0.058 & 18.65 & 2.64 & 3.74 & 51 & 0.050 & 26.57 & 3.25 & 4.56 & & \\
\hline & Normal-weight & 34 & 0.063 & 29.79 & 3.85 & 5.78 & 31 & 0.058 & 18.65 & 3.06 & 4.33 & 33 & 0.050 & 8.91 & 2.50 & 2.01 & \multirow{2}{*}{$\mathrm{TxW}(p=0.045)$} & \\
\hline & Overweight/obese & 17 & 0.200 & 56.35 & $8.62^{\mathrm{a}}$ & 15.14 & 17 & 0.063 & 8.38 & $1.87^{\mathrm{b}}$ & 2.24 & 18 & 0.079 & 26.57 & $4.62^{\mathrm{ab}}$ & 7.11 & & \\
\hline \multirow{3}{*}{ miR-17 } & Whole & 52 & 0.047 & 34.02 & $4.88^{\mathrm{a}}$ & 6.39 & 52 & 0.083 & 17.68 & $2.55^{\mathrm{b}}$ & 3.31 & 55 & 0.069 & 17.34 & $2.95^{\mathrm{b}}$ & 3.42 & $\mathrm{~T}(p=0.029)$ & \\
\hline & Normal-wei & 34 & 0.047 & 21.32 & 4.01 & 4.50 & 34 & 0.083 & 17.68 & 2.94 & 3.79 & 36 & 0.126 & 13.37 & 2.78 & 2.74 & \multirow{2}{*}{$\mathrm{TxW}(p=0.034)$} & \\
\hline & Overweight/obese & 18 & 0.144 & 34.02 & $6.52^{a}$ & 8.89 & 18 & 0.180 & 6.48 & $1.80^{\mathrm{b}}$ & 2.01 & 19 & 0.069 & 17.34 & $3.27 \mathrm{ab}$ & 4.50 & & $\mathrm{~T}(p=0.048)$ \\
\hline
\end{tabular}


Table 1. Cont.

\begin{tabular}{|c|c|c|c|c|c|c|c|c|c|c|c|c|c|c|c|c|c|c|}
\hline & & \multicolumn{5}{|c|}{ Month 1} & \multicolumn{5}{|c|}{ Month 2} & \multicolumn{5}{|c|}{ Month 3} & \multicolumn{2}{|c|}{ Statistical Analysis } \\
\hline & & $\mathbf{N}$ & Min & $\operatorname{Max}$ & Mean & SD & $\mathbf{N}$ & Min & $\operatorname{Max}$ & Mean & SD & $\mathbf{N}$ & Min & $\operatorname{Max}$ & Mean & SD & Overall & $\begin{array}{c}\text { Normal/Obese } \\
\text { Weight }\end{array}$ \\
\hline \multirow{3}{*}{ miR-let7b } & Whole & 52 & 0.157 & 244.25 & 26.50 & 41.50 & 54 & 0.316 & 85.16 & 14.79 & 17.44 & 55 & 0.584 & 89.15 & 16.86 & 19.25 & & \\
\hline & Normal-weight & 34 & 0.157 & 93.52 & 19.64 & 19.91 & 36 & 0.316 & 85.16 & 16.77 & 19.66 & 36 & 0.584 & 89.15 & 15.94 & 16.30 & & \\
\hline & Overweight/obese & 18 & 0.659 & 244.25 & 39.47 & 64.23 & 18 & 0.773 & 39.07 & 10.83 & 11.30 & 19 & 0.714 & 83.21 & 18.60 & 24.30 & & \\
\hline \multirow[b]{2}{*}{ miR-let7c } & Whole & 51 & 0.035 & 23.88 & $2.88^{\mathrm{a}}$ & 4.54 & 54 & 0.024 & 9.21 & $1.37^{\mathrm{ab}}$ & 1.76 & 55 & 0.053 & 7.04 & $1.27^{\mathrm{b}}$ & 1.48 & $\mathrm{~T}(p=0.014)$ & \\
\hline & $\begin{array}{l}\text { Normal-weight } \\
\text { Overweight/obese }\end{array}$ & $\begin{array}{l}33 \\
18\end{array}$ & 0.035 & $\begin{array}{c}9.31 \\
23.88\end{array}$ & $\begin{array}{r}2.10 \\
4.32^{\mathrm{a}}\end{array}$ & $\begin{array}{l}1.97 \\
7.07\end{array}$ & 36 & 0.024 & $\begin{array}{l}6.70 \\
9.21\end{array}$ & $\begin{array}{c}1.41 \\
1.30^{\mathrm{a}, \mathrm{b}}\end{array}$ & $\begin{array}{l}1.62 \\
2.07\end{array}$ & 36 & 0.053 & $\begin{array}{l}5.85 \\
7.04\end{array}$ & $\begin{array}{r}1.19 \\
1.44^{\mathrm{b}}\end{array}$ & 1.12 & $\mathrm{~T}(p=0.016)$ & \\
\hline \multirow{3}{*}{ miR-146b } & Whole & 50 & 0.148 & 354.36 & $35.70^{\mathrm{a}}$ & 65.49 & 48 & 0.427 & 62.18 & $12.02^{\mathrm{b}}$ & 12.85 & 53 & 0.034 & 78.81 & $14.77^{\mathrm{b}}$ & 17.66 & $\mathrm{~T}(p=0.008)$ & \\
\hline & Normal-weight & 32 & 0.148 & 103.03 & $24.10^{\mathrm{a}}$ & 29.06 & 31 & 0.448 & 62.18 & $13.26^{\mathrm{b}}$ & 13.15 & 34 & 0.034 & 44.90 & $13.32^{b}$ & 12.60 & \multirow{2}{*}{$\mathrm{T}(p=0.001)$} & $\mathrm{T}(p=0.029)$ \\
\hline & Overweight/obese & 18 & 0.890 & 354.36 & $56.31^{\mathrm{a}}$ & 100.61 & 17 & 0.427 & 40.83 & $9.75^{\mathrm{b}}$ & 12.33 & 19 & 0.090 & 78.81 & $17.37^{\mathrm{b}}$ & 24.48 & & $\mathrm{~T}(p=0.027)$ \\
\hline
\end{tabular}

Breast milk miRNAs were purified with the microRNA isolation kit mirVana and determined in breast milk at month 1, 2, and 3 of lactation by TaqMan microRNA specific assays. Whole population was segregated at sampling time into normal-weight $(\mathrm{BMI}<25)$ and overweight/obese $(\mathrm{BMI} \geq 25)$ mothers. Repeated measures analysis of variance $(\mathrm{rANOVA})$ was used to assess the impact of time of lactation (T) on the whole population, and then to explore the influence of maternal overweight/obesity (W) throughout lactation (T). This was followed by pairwise post-hoc comparison to detect differences between months. Statistically significant differences were considered when $p<0.05$, and are indicated in the columns overall and normal/obese-weight; in addition, significances that appeared by pair comparison are represented in data not sharing the same superscript (in the same row). $\mathrm{N}$ (number of subjects); Min (minimum); Max (maximum); SD (standard deviation). The miRNAs studied are described as miR-followed by their specific code. 
Table 2. Breast milk leptin and adiponectin.

\begin{tabular}{|c|c|c|c|c|c|c|c|c|c|c|c|c|c|c|c|c|c|c|}
\hline & & \multicolumn{5}{|c|}{ Month 1} & \multicolumn{5}{|c|}{ Month 2} & \multicolumn{5}{|c|}{ Month 3} & \multicolumn{2}{|c|}{ Statistical Analysis } \\
\hline & & $\mathbf{N}$ & Min & $\operatorname{Max}$ & Mean & SD & $\mathbf{N}$ & Min & $\operatorname{Max}$ & Mean & SD & $\mathbf{N}$ & Min & $\operatorname{Max}$ & Mean & SD & Overall & $\begin{array}{c}\text { Normal/Obese } \\
\text { Weight }\end{array}$ \\
\hline \multirow{3}{*}{$\begin{array}{l}\text { Leptin } \\
(\mathrm{ng} / \mathrm{mL})\end{array}$} & Whole & 53 & 0.020 & 1.92 & $0.376^{\mathrm{a}}$ & 0.353 & 55 & 0.003 & 1.18 & $0.341^{\mathrm{b}}$ & 0.314 & 54 & 0.003 & 1.45 & $0.346^{\mathrm{b}}$ & 0.333 & $\begin{array}{c}\text { Normal/Obese } \\
\text { weight }\end{array}$ & \multirow{3}{*}{$\mathrm{T}(p=0.010)$} \\
\hline & Normal-weight & 35 & 0.020 & 0.60 & $0.235^{a}$ & 0.153 & 36 & 0.003 & 0.98 & $0.242^{b}$ & 0.242 & 35 & 0.020 & 1.08 & $0.209^{b}$ & 0.195 & $\mathrm{~T}(p=0.030)$ & \\
\hline & Overweight/obese & 18 & 0.156 & 1.92 & 0.652 & 0.462 & 19 & 0.020 & 1.18 & 0.529 & 0.355 & 19 & 0.003 & 1.45 & 0.597 & 0.388 & $\mathrm{~W}^{\prime}(p=0.000)$ & \\
\hline \multirow{3}{*}{$\begin{array}{l}\text { Adiponectin } \\
\text { (ng/mL) }\end{array}$} & Whole & 53 & 8.90 & 48.50 & 23.39 & 7.57 & 55 & 7.30 & 44.10 & 20.83 & 6.61 & 53 & 9.30 & 65.70 & 20.32 & 8.02 & & \multirow{3}{*}{$\mathrm{T}(p=0.000)$} \\
\hline & Normal-weight & 35 & 10.70 & 48.50 & $23.16^{\mathrm{a}}$ & 7.97 & 36 & 7.30 & 44.10 & $19.19^{\mathrm{b}}$ & 7.03 & 35 & 9.30 & 26.80 & $18.81^{\mathrm{b}}$ & 4.62 & \multirow{2}{*}{$\mathrm{TxW}(p=0.035)$} & \\
\hline & Overweight/obese & 18 & 8.90 & 34.00 & 23.03 & 6.94 & 19 & 10.00 & 31.50 & 22.37 & 5.58 & 18 & 13.30 & 65.70 & 23.38 & 11.80 & & \\
\hline
\end{tabular}

Breast milk leptin and adiponectin were determined by ELISA at month 1, 2, and 3 of lactation. Whole population was segregated at sampling time into normal-weight (BMI $<25)$ and overweight/obese (BMI $\geq 25)$ mothers. Repeated measures analysis of variance (rANOVA) was used to assess the impact of time of lactation (T) on the whole population, and then to explore the influence of maternal overweight/obesity (W) throughout lactation (T), which was followed by pairwise post-hoc comparison to detect differences between months. Statistically significant differences were considered when $p<0.05$ and are indicated in the respective columns of overall and normal/obese-weight; in addition, significances that appeared by pair comparison are represented in data not sharing the same superscript (in the same row). N (number of subjects); Min (minimum); Max (maximum); SD (standard deviation). 
Table 3. Correlations between milk leptin, adiponectin and miRNAs at the second month of lactation and their relationship with infant BMI at 2 years of age.

\begin{tabular}{|c|c|c|c|c|c|c|c|c|c|c|c|c|c|c|c|c|}
\hline & & & Adiponectin & miR-30a & miR-27a & $\operatorname{miR}-27 \mathrm{~b}$ & miR-148a & miR-let7a & miR-let7b & miR-103 & miR-17 & miR-181a & miR-222 & miR-let7c & miR-146b & miR-200b \\
\hline \multirow{6}{*}{ Leptin } & \multirow{2}{*}{ Whole } & 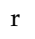 & 0.214 & -0.195 & -0.092 & -0.165 & -0.064 & -0.146 & -0.151 & $-0.325^{*}$ & -0.198 & -0.172 & -0.162 & -0.110 & -0.263 & -0.184 \\
\hline & & $\mathrm{p}$ & 0.117 & 0.161 & 0.508 & 0.233 & 0.647 & 0.322 & 0.275 & 0.016 & 0.160 & 0.214 & 0.246 & 0.428 & 0.071 & 0.206 \\
\hline & \multirow{2}{*}{ Normal-weight } & $\mathrm{r}$ & 0.089 & $-0.454^{* *}$ & $-0.380^{*}$ & $-0.410^{*}$ & -0.150 & -0.328 & -0.276 & $-0.574^{* *}$ & $-0.419^{*}$ & $-0.470^{* *}$ & $-0.471^{* *}$ & -0.261 & $-0.473^{* *}$ & -0.297 \\
\hline & & $\mathrm{p}$ & 0.605 & 0.006 & 0.022 & 0.013 & 0.381 & 0.072 & 0.103 & 0.000 & 0.014 & 0.004 & 0.004 & 0.125 & 0.007 & 0.098 \\
\hline & \multirow{2}{*}{\multicolumn{2}{|c|}{ Overweight/Obese ${ }_{\mathrm{p}}^{\mathrm{r}}$}} & 0.136 & 0.159 & 0.352 & 0.180 & 0.112 & 0.170 & 0.248 & 0.114 & 0.301 & 0.267 & 0.386 & 0.214 & 0.139 & 0.055 \\
\hline & & & 0.580 & 0.529 & 0.152 & 0.476 & 0.670 & 0.513 & 0.321 & 0.654 & 0.224 & 0.283 & 0.113 & 0.394 & 0.596 & 0.833 \\
\hline \multirow{6}{*}{ Adiponectin } & \multirow{2}{*}{ Whole } & $\mathrm{r}$ & 1,000 & $-0.345^{*}$ & -0.123 & -0.198 & $-0.353^{* *}$ & $-0.374^{* *}$ & $-0.403^{* *}$ & $-0.365^{* *}$ & $-0.425^{* *}$ & $-0.275^{*}$ & $-0.310^{*}$ & $-0.329^{*}$ & $-0.293^{*}$ & -0.248 \\
\hline & & $\mathrm{p}$ & & 0.011 & 0. & 0.152 & 0.010 & 0.009 & 0.003 & 0.007 & 0.002 & 0.044 & 0.024 & 0.015 & 0.043 & 0.086 \\
\hline & \multirow{2}{*}{ Normal-weight } & $\mathrm{r}$ & 1,000 & -0.325 & -0.071 & -0.134 & -0.388 * & $-0.467^{* *}$ & $-0.384^{*}$ & $-0.393^{*}$ & $-0.418 *$ & -0.204 & -0.299 & -0.319 & -0.240 & -0.207 \\
\hline & & $\mathrm{p}$ & & 0.057 & 0.682 & 0.435 & 0.019 & 0.008 & 0.021 & 0.018 & 0.014 & 0.232 & 0.081 & 0.058 & 0.193 & 0.255 \\
\hline & \multirow{2}{*}{ Overweight/Obese } & $\mathrm{r}$ & 1,000 & -0.209 & -0.184 & -0.291 & -0.222 & -0.006 & -0.151 & -0.180 & -0.250 & -0.164 & -0.163 & -0.313 & -0.085 & -0.098 \\
\hline & & $\mathrm{p}$ & & 0.406 & 0.465 & 0.241 & 0.392 & 0.981 & 0.550 & 0.476 & 0.317 & 0.515 & 0.518 & 0.206 & 0.747 & 0.708 \\
\hline \multirow{6}{*}{ BMI24 } & \multirow[b]{2}{*}{ Whole } & $\mathrm{r}$ & 0.12 & -0.246 & -0.167 & -0.218 & -0.252 & $-0.326^{*}$ & -0.278 & $-0.425^{* *}$ & $-0.298^{*}$ & -0.238 & $-0.298^{*}$ & $-0.331^{*}$ & -0.287 & $-0.347^{*}$ \\
\hline & & $\mathrm{p}$ & 0.380 & 0.085 & 0.245 & 0.129 & 0.081 & 0.031 & 0.051 & 0.002 & 0.040 & 0.096 & 0.037 & 0.019 & 0.059 & 0.020 \\
\hline & \multirow{2}{*}{ Normal-weight } & $\mathrm{r}$ & 0.199 & -0.302 & -0.196 & -0.276 & -0.207 & -0.296 & -0.313 & $-0.521^{* *}$ & $-0.427^{*}$ & $-0.347^{*}$ & $-0.385^{*}$ & $-0.360^{*}$ & $-0.422^{*}$ & -0.327 \\
\hline & & $\mathrm{p}$ & 0.260 & 0.082 & 0.267 & 0.114 & 0.239 & 0.120 & 0.071 & 0.002 & 0.015 & 0.044 & 0.027 & 0.036 & 0.023 & 0.078 \\
\hline & \multirow{2}{*}{ Overweight/Obese } & $\mathrm{r}$ & -0.124 & -0.224 & -0.179 & -0.071 & -0.377 & -0.379 & -0.285 & -0.265 & -0.171 & -0.106 & -0.138 & -0.276 & -0.218 & -0.318 \\
\hline & & $\mathrm{p}$ & 0.636 & 0.405 & 0.506 & 0.795 & 0.166 & 0.164 & 0.284 & 0.322 & 0.528 & 0.696 & 0.610 & 0.300 & 0.435 & 0.248 \\
\hline
\end{tabular}

Whole population was segregated at sampling time into normal-weight mothers (BMI < 25) and overweight/obese (BMI $\geq 25)$. Associations between milk leptin, adiponectin, and miRNAs in breast milk at the second month of lactation and with infant BMI at 24 months of age (BMI24) were tested by Spearman's correlation test. Spearman's rank correlation coefficient (r) and significance $(p)$ are shown. ${ }^{* *}=p<0.01$ (bilateral); ${ }^{*}=p<0.05$ (bilateral). The miRNAs studied are described as miR-followed by their specific code. 


\section{Discussion}

We tested the hypothesis that the presence of breast milk miRNAs could be altered in overweight/obese lactating mothers, and there could be a potential relationship with leptin and adiponectin functions in breast milk. Data showed that the supply of miRNAs in maternal milk was altered in overweight/obesity. Furthermore, the associations found between milk miRNAs, leptin, adiponectin, and infant weight were also disturbed in the offspring from overweight or obese mothers.

The study of miRNAs in breast milk is a topic of great interest in the field of nutrition given their potential involvement in infant development and future health. The relatively immature gastrointestinal tract of newborns may allow effective molecular communication between mother and infant through miRNAs, as suggested by the presence of immune-related miRNAs in mammal milk [2]. Furthermore, growing evidence indicates that miRNAs expressed in breast milk may reflect maternal diet and nutritional status and, therefore, may influence offspring phenotype $[8,28]$.

Human milk is a rich source of lactation-specific miRNAs, which could be used as biomarkers of the performance and health status of both the lactating mammary gland and the breastfed infant $[29,30]$. Maternal obesity is a factor that may influence miRNA content and expression levels, as has been shown in plasma [9] and placenta [31] during perinatal development. However, the influence of overweight/obesity on the role of miRNAs supplied during breastfeeding is unknown. Here, our approach was to focus on a set of miRNAs with a potential link with obesity and the key regulators leptin and adiponectin, bearing in mind the important role of these proteins in breast milk, particularly leptin, as determinants of a healthy metabolic programming for life as recently revised [15]. Thus, expression and regulation throughout lactation of miRNA genes that are putative targets of leptin (miR-146b, miR-27a, miR-27b, miR-17, miR-30a), adiponectin (miR-181a, miR-27a, miR-27b), and their receptors (miR-let 7a, miR-let7b, miR-let7c, miR-222, miR-30a) or related to adipogenesis and obesity (miR-103, miR-17) were characterized in overweight/obese lactating mothers in comparison with normal-weight mothers. Interestingly, expression of miRNAs associated with obesity was shown (e.g., miR-221) together with others miRNAs previously described as milk-derived miRNAs [32] (e.g., miR-let7a and miR-let7b). In addition, other tissue-specific miRNAs (e.g., miR-95, miR-451) [11,21] could not be detected in breast milk, suggesting a mammary gland-specific delivery of miRNAs to the newborn.

High variability regarding milk miRNAs content was found between women, as seen in previous studies [2,12]. Data in the normal-weight group were consistent with a smooth decline in the miRNA levels analyzed throughout the first three months of lactation, with the exception of miR-30a, which increased over time. In general terms, our data would fit with the few reports that have analyzed the time course of miRNAs in human breast milk and describe higher levels in early milk, particularly in colostrum, compared with mature milk [2,12]. In contrast, overweight/obese mothers tended to show higher levels during the first month and then sharper decrease in the following months (miR-222, miR-103, miR-17, miR-146b). A number of miRNAs have been linked with obesity or dietary profile $[9,33,34]$, but data on breast milk miRNAs in overweight/obese women as obtained in the present study are lacking.

Intriguingly, the genes targeted by the six human milk miRNAs that showed altered profiles over time due to overweight/maternal obesity (miR-let7a, miR-103, miR-222, miR-17, miR-146b, miR-30a) were found to be involved in a set of pathways which are very relevant in the developmental process. In this regard, enriched transcription related gene ontology biological processes were connected to three main features: processes involving cell development, morphogenesis and differentiation; a node specifically dealing with neurodevelopment; and another with miRNAs delivery (lipid particle organization and vesicle transport). Involvement of milk miRNAs in infant brain development has already been suggested in preterm delivery [35]. The fact that miRNAs may also be involved in mammary gland signaling during lactation has been shown in dairy cows [36] and humans [37]. All the above points to the fact that milk-miRNAs may influence the growth and development of the newborn, and that maternal overweight/obesity would result in different outcomes from normal-weight 
mothers. Although we cannot establish a direct cause-effect relationship, it can be hypothesized that in normal weight mothers miRNAs profile in breast milk could modulate metabolic programing in the offspring [38] and this effect can be altered in the case of overweight/obesity.

Human milk miRNAs are found in breast milk fat globules or enriched in the milk lipid fraction [13,39]. Our analysis was performed in whole milk to ensure total extraction of the miRNAs of interest. To assess the relevance of milk fat on miRNA content, correlation analyses were undertaken. Interestingly, levels of miRNAs in normal-weight mothers were negatively associated with MUFA at the first month of lactation, positively associated with saturated fatty acid content at the second month, and positively associated with triglycerides at the third month. This pattern, absent in overweight/obese women, would suggest a strong dependency of breast milk miRNAs on the quality of the milk fat content, which is influenced by diet [40]. Future studies assessing the impact of dietary fat on breast milk miRNAs and fatty acid content would be necessary to fully understand the secretion of breast milk miRNAs and their regulation. Furthermore, this knowledge could be taken into consideration when designing dietary interventions to optimize breast milk miRNAs in the future.

Breastfeeding is protective against obesity in adult life [41-43], which has been attributed to bioactive compounds that are present in breast milk, but absent in infant formula. Leptin, a main regulator of energy balance, has a key preventive role of obesity during lactation [14,16,44-46]. A number of reports have shown a positive correlation between milk leptin and maternal adiposity [47] and a negative association between breast milk leptin, particularly in normal-weight women, and infant weight gain $[16,17,46,48]$. Evidence recorded in two recent meta-analyses reached the conclusion that breastfeeding is a protective factor against childhood obesity [42,43]. Furthermore, proof that suckling animals supplemented with leptin have a decreased food intake, show healthier food preferences, and are protected against overweight in adulthood, mediated by mechanisms associated with leptin and insulin sensitivity, are critical findings to understand the underlying basis of early human obesity programming [17,19,45,49-51].

Leptin supply is highly variable, and the main factor conditioning breast milk leptin secretion is the content of maternal body fat stores [47]. Therefore, newborn infants breast fed by obese mothers are exposed to a higher amount of milk leptin; but contrary to expectations, they are more likely to become obese [52], as due to missing the leptin protection role. Further studies are needed to elucidate whether specific milk-derived miRNAs, such as those differentially expressed in overweight/obese in comparison with normal-weight mothers, could be involved in the mechanisms underlying a loss of leptin functionality in the case of obesity.

In contrast with leptin, the role of adiponectin affecting metabolic regulation and programming of developing infants is less known, but it has been described that higher milk levels could be associated with greater adiposity in offspring [44]. In this context, our data give shape to the idea that a set of miRNAs present in breast milk may act, in close conjunction with leptin and adiponectin, as bioactive compounds with the potential to modulate infant body weight and composition (Figure 3). Potentially, common modulatory mechanisms of secretion in the mammary gland could be responsible for this association, which remains to be tested. Our data are suggestive of an interplay between leptin, adiponectin and a core of miRNAs in milk, which in the end modulate the BMI of infants at 2 years of age. Furthermore, it is apparent that maternal overweight/obesity alters this interplay. To gain a better understanding of the mechanism of action would enable better strategies in the prevention of obesity, including better nutrition of mothers to optimize milk composition and, when breastfeeding is not possible, this knowledge would potentially contribute to the improvement of infant formula to support optimized growth. 


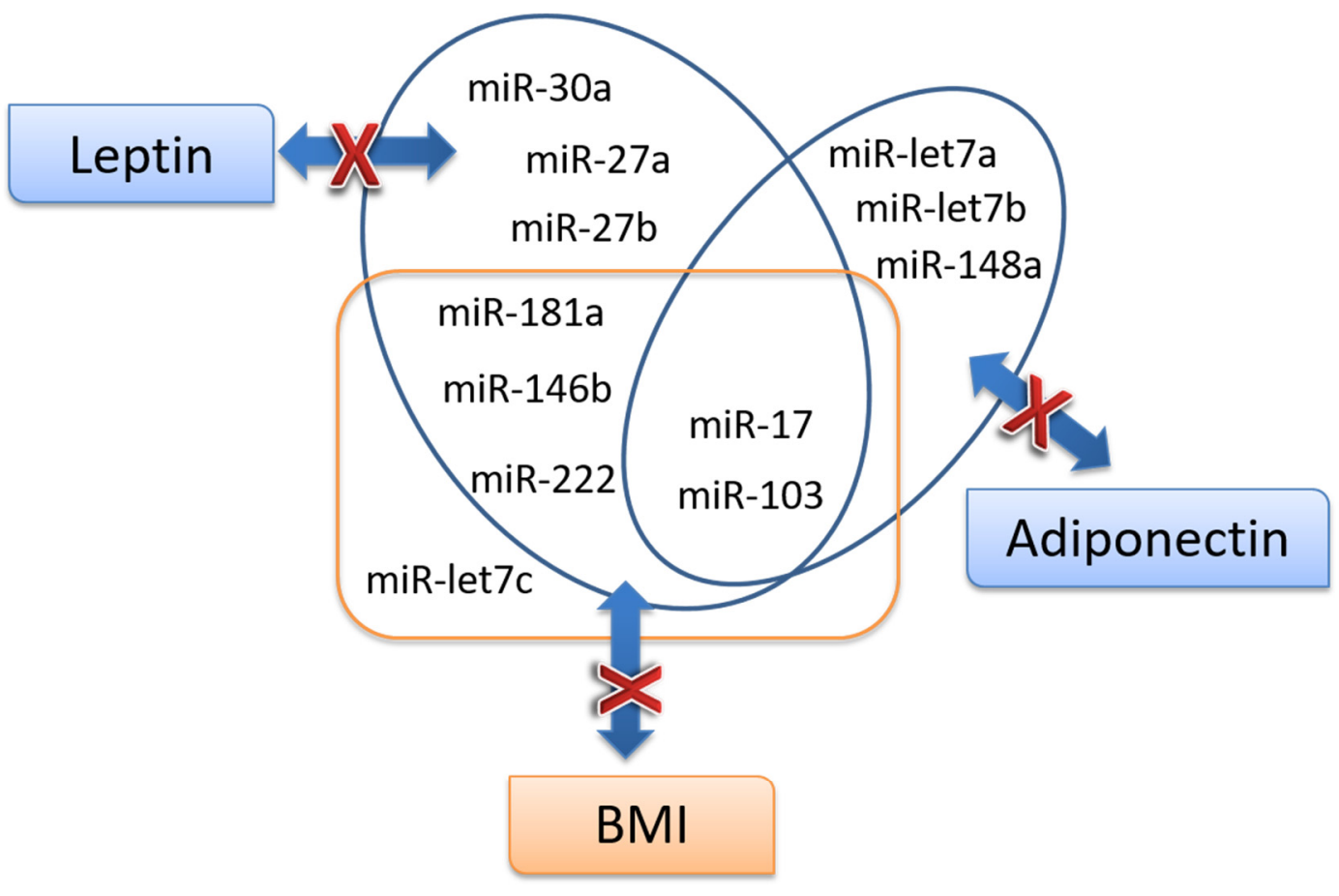

\section{$\Rightarrow$ Negative correlation found in normal-weight mothers No correlation found in overweight/obese mothers}

Figure 3. Interactions observed with milk miRNAs, leptin, and adiponectin and their relationship with infant BMI. Schematic representation of the correlations observed between milk miRNAs and leptin, adiponectin, and infant BMI. Milk data are from the second month of lactation and infant BMI corresponds to data collected at 24 months of age. These relationships were observed in normal-weight mothers but were absent in overweight/obesity.

Supplementary Materials: The following are available online at http://www.mdpi.com/2072-6643/11/11/2589/s1, Table S1. miRNAs selected to assay in breast milk from lactating mothers. Table S2. Participant characteristics. Table S3. Maternal data and infant anthropometry. Table S4. Correlations between miRNAs during lactation and fatty acid content in milk.

Author Contributions: F.S. and A.P. designed the study. R.Z. was responsible for cohort recruitment and follow-up, data collection, laboratory analysis and elaboration of the results database. R.Z. prepared a first draft of the manuscript. F.S. performed the statistical analysis. J.S. performed the in-silico analysis and contributed to the discussion. F.S. and A.P. wrote the final version of the manuscript. All authors approved the final version of the submitted manuscript.

Funding: This research was funded in part by grants: AGL2015-67019-P (Agencia Estatal de Investigación, MINECO/FEDER; EU), PGC2018-097436-B-I00 (MCIU/AEI/FEDER, UE) and, Project PI17/01614, funded by Instituto de Salud Carlos III and co-funded by European Union (ERDF/ESF, "Investing in your future"). Our group receives financial support from Instituto de Salud Carlos III, Centro de Investigación Biomédica en Red Fisiopatología de la Obesidad y Nutrición, CIBERobn.

Acknowledgments: We thank the women and infants who participated in the study. We acknowledge the support obtained from the Center of 'Atenció Primaria de Mallorca' through the 'Centro de Salud' of Manacor and Palma (S'Escorxador and Son Ferriol). We appreciate the support and engagement of the midwives J. Amengual, M. Maimó and M.C. Carrascosa, who supported mothers during the lactation period and contributed to maternal follow-up until the end of the study.

Conflicts of Interest: The authors declare no conflict of interest. 


\section{References}

1. Woo Baidal, J.A.; Locks, L.M.; Cheng, E.R.; Blake-Lamb, T.L.; Perkins, M.E.; Taveras, E.M. Risk factors for childhood obesity in the first 1000 days: A systematic review. Am. J. Prev. Med. 2016, 50, 761-779. [CrossRef] [PubMed]

2. Kosaka, N.; Izumi, H.; Sekine, K.; Ochiya, T. MicroRNA as a new immune-regulatory agent in breast milk. Silence 2010, 1, 7. [CrossRef] [PubMed]

3. Na, R.S.; E, G.X.; Sun, W.; Sun, X.W.; Qiu, X.Y.; Chen, L.P.; Huang, Y.F. Expressional analysis of immune-related miRNAs in breast milk. Genet. Mol. Res. 2015, 14, 11371-11376. [CrossRef] [PubMed]

4. Zhou, Q.; Li, M.; Wang, X.; Li, Q.; Wang, T.; Zhu, Q.; Zhou, X.; Gao, X.; Li, X. Immune-related microRNAs are abundant in breast milk exosomes. Int. J. Biol. Sci. 2012, 8, 118-123. [CrossRef] [PubMed]

5. Alsaweed, M.; Lai, C.T.; Hartmann, P.E.; Geddes, D.T.; Kakulas, F. Human milk cells and lipids conserve numerous known and novel miRNAs, some of which are differentially expressed during lactation. PLoS ONE 2016, 11, e0152610. [CrossRef]

6. Benmoussa, A.; Lee, C.H.; Laffont, B.; Savard, P.; Laugier, J.; Boilard, E.; Gilbert, C.; Fliss, I.; Provost, P. Commercial dairy cow milk microRNAs resist digestion under simulated gastrointestinal tract conditions. J. Nutr. 2016, 146, 2206-2215. [CrossRef]

7. Izumi, H.; Kosaka, N.; Shimizu, T.; Sekine, K.; Ochiya, T.; Takase, M. Bovine milk contains microRNA and messenger RNA that are stable under degradative conditions. J. Dairy Sci. 2012, 95, 4831-4841. [CrossRef]

8. Pomar, C.A.; Castro, H.; Pico, C.; Serra, F.; Palou, A.; Sanchez, J. Cafeteria diet consumption during lactation in rats, rather than obesity Per Se, alters miR-222, miR-200a, and miR-26a levels in milk. Mol. Nutr. Food Res. 2019, 63, e1800928. [CrossRef]

9. Carreras-Badosa, G.; Bonmati, A.; Ortega, F.J.; Mercader, J.M.; Guindo-Martinez, M.; Torrents, D.; Prats-Puig, A.; Martinez-Calcerrada, J.M.; Platero-Gutierrez, E.; De Zegher, F.; et al. Altered circulating miRNA expression profile in pregestational and gestational obesity. J. Clin. Endocrinol. Metab. 2015, 100, E1446-E1456. [CrossRef]

10. Can, U.; Buyukinan, M.; Yerlikaya, F.H. The investigation of circulating microRNAs associated with lipid metabolism in childhood obesity. Pediatr. Obes. 2016, 11, 228-234. [CrossRef]

11. Prats-Puig, A.; Ortega, F.J.; Mercader, J.M.; Moreno-Navarrete, J.M.; Moreno, M.; Bonet, N.; Ricart, W.; Lopez-Bermejo, A.; Fernandez-Real, J.M. Changes in circulating microRNAs are associated with childhood obesity. J. Clin. Endocrinol. Metab. 2013, 98, E1655-E1660. [CrossRef]

12. Xi, Y.; Jiang, X.; Li, R.; Chen, M.; Song, W.; Li, X. The levels of human milk microRNAs and their association with maternal weight characteristics. Eur. J. Clin. Nutr. 2016, 70, 445-449. [CrossRef] [PubMed]

13. Munch, E.M.; Harris, R.A.; Mohammad, M.; Benham, A.L.; Pejerrey, S.M.; Showalter, L.; Hu, M.; Shope, C.D.; Maningat, P.D.; Gunaratne, P.H.; et al. Transcriptome profiling of microRNA by Next-Gen deep sequencing reveals known and novel miRNA species in the lipid fraction of human breast milk. PLoS ONE 2013, 8, e50564. [CrossRef] [PubMed]

14. Palou, A.; Sanchez, J.; Pico, C. Nutrient-gene interactions in early life programming: Leptin in breast milk prevents obesity later on in life. Adv. Exp. Med. Biol. 2009, 646, 95-104. [CrossRef] [PubMed]

15. Palou, M.; Pico, C.; Palou, A. Leptin as a breast milk component for the prevention of obesity. Nutr. Rev. 2018, 76, 875-892. [CrossRef]

16. Miralles, O.; Sanchez, J.; Palou, A.; Pico, C. A physiological role of breast milk leptin in body weight control in developing infants. Obesity (Silver Spring) 2006, 14, 1371-1377. [CrossRef]

17. Pico, C.; Oliver, P.; Sanchez, J.; Miralles, O.; Caimari, A.; Priego, T.; Palou, A. The intake of physiological doses of leptin during lactation in rats prevents obesity in later life. Int. J. Obes. (Lond) 2007, 31, 1199-1209. [CrossRef]

18. Pico, C.; Palou, M.; Priego, T.; Sanchez, J.; Palou, A. Metabolic programming of obesity by energy restriction during the perinatal period: Different outcomes depending on gender and period, type and severity of restriction. Front. Physiol. 2012, 3, 436. [CrossRef]

19. Sanchez, J.; Priego, T.; Palou, M.; Tobaruela, A.; Palou, A.; Pico, C. Oral supplementation with physiological doses of leptin during lactation in rats improves insulin sensitivity and affects food preferences later in life. Endocrinology 2008, 149, 733-740. [CrossRef] 
20. Chen, X.; Gao, C.; Li, H.; Huang, L.; Sun, Q.; Dong, Y.; Tian, C.; Gao, S.; Dong, H.; Guan, D.; et al. Identification and characterization of microRNAs in raw milk during different periods of lactation, commercial fluid, and powdered milk products. Cell Res. 2010, 20, 1128-1137. [CrossRef]

21. Bao, B.; Rodriguez-Melendez, R.; Wijeratne, S.S.; Zempleni, J. Biotin regulates the expression of holocarboxylase synthetase in the miR-539 pathway in HEK-293 cells. J. Nutr. 2010, 140, 1546-1551. [CrossRef] [PubMed]

22. Friedman, Y.; Naamati, G.; Linial, M. MiRror: A combinatorial analysis web tool for ensembles of microRNAs and their targets. Bioinformatics 2010, 26, 1920-1921. [CrossRef] [PubMed]

23. Bindea, G.; Galon, J.; Mlecnik, B. CluePedia Cytoscape plugin: Pathway insights using integrated experimental and in silico data. Bioinformatics 2013, 29, 661-663. [CrossRef] [PubMed]

24. Bindea, G.; Mlecnik, B.; Hackl, H.; Charoentong, P.; Tosolini, M.; Kirilovsky, A.; Fridman, W.H.; Pages, F.; Trajanoski, Z.; Galon, J. ClueGO: A Cytoscape plug-in to decipher functionally grouped gene ontology and pathway annotation networks. Bioinformatics 2009, 25, 1091-1093. [CrossRef] [PubMed]

25. Shannon, P.; Markiel, A.; Ozier, O.; Baliga, N.S.; Wang, J.T.; Ramage, D.; Amin, N.; Schwikowski, B.; Ideker, T. Cytoscape: A software environment for integrated models of biomolecular interaction networks. Genome Res. 2003, 13, 2498-2504. [CrossRef]

26. Laraichi, S.; Parra, P.; Zamanillo, R.; El Amarti, A.; Palou, A.; Serra, F. Dietary supplementation of calcium may counteract obesity in mice mediated by changes in plasma fatty acids. Lipids 2013, 48, 817-826. [CrossRef]

27. Ley, S.H.; Hanley, A.J.; Stone, D.; O'Connor, D.L. Effects of pasteurization on adiponectin and insulin concentrations in donor human milk. Pediatr. Res. 2011, 70, 278-281. [CrossRef]

28. Zempleni, J.; Aguilar-Lozano, A.; Sadri, M.; Sukreet, S.; Manca, S.; Wu, D.; Zhou, F.; Mutai, E. Biological activities of Extracellular Vesicles and their cargos from bovine and human milk in humans and implications for infants. J. Nutr. 2017, 147, 3-10. [CrossRef]

29. Alsaweed, M.; Lai, C.T.; Hartmann, P.E.; Geddes, D.T.; Kakulas, F. Human milk miRNAs primarily originate from the mammary gland resulting in unique miRNA profiles of fractionated milk. Sci. Rep. 2016, 6, 20680. [CrossRef]

30. Pico, C.; Serra, F.; Rodriguez, A.M.; Keijer, J.; Palou, A. Biomarkers of nutrition and health: New tools for new approaches. Nutrients 2019, 11, 1092. [CrossRef]

31. Carreras-Badosa, G.; Bonmati, A.; Ortega, F.J.; Mercader, J.M.; Guindo-Martinez, M.; Torrents, D.; Prats-Puig, A.; Martinez-Calcerrada, J.M.; de Zegher, F.; Ibanez, L.; et al. Dysregulation of placental miRNA in maternal obesity is associated with Pre- and postnatal growth. J. Clin. Endocrinol. Metab. 2017, 102, 2584-2594. [CrossRef] [PubMed]

32. Melnik, B.C.; John, S.M.; Schmitz, G. Milk is not just food but most likely a genetic transfection system activating mTORC1 signaling for postnatal growth. Nutr. J. 2013, 12, 103. [CrossRef] [PubMed]

33. McGregor, R.A.; Choi, M.S. MicroRNAs in the regulation of adipogenesis and obesity. Curr. Mol. Med. 2011, 11, 304-316. [CrossRef] [PubMed]

34. Parra, P.; Serra, F.; Palou, A. Expression of adipose microRNAs is sensitive to dietary conjugated linoleic acid treatment in mice. PLoS ONE 2010, 5, e13005. [CrossRef] [PubMed]

35. Carney, M.C.; Tarasiuk, A.; DiAngelo, S.L.; Silveyra, P.; Podany, A.; Birch, L.L.; Paul, I.M.; Kelleher, S.; Hicks, S.D. Metabolism-related microRNAs in maternal breast milk are influenced by premature delivery. Pediatr. Res. 2017, 82, 226-236. [CrossRef]

36. Do, D.N.; Li, R.; Dudemaine, P.L.; Ibeagha-Awemu, E.M. MicroRNA roles in signalling during lactation: An insight from differential expression, time course and pathway analyses of deep sequence data. Sci. Rep. 2017, 7, 44605. [CrossRef]

37. Alsaweed, M.; Hartmann, P.E.; Geddes, D.T.; Kakulas, F. MicroRNAs in breastmilk and the lactating Breast: potential immunoprotectors and developmental regulators for the infant and the mother. Int. J. Environ. Res. Public Health 2015, 12, 13981-14020. [CrossRef]

38. Lonnerdal, B. Human milk microRNAs/exosomes: Composition and biological effects. Nestle Nutr. Inst. Workshop Ser. 2019, 90, 83-92. [CrossRef]

39. Alsaweed, M.; Hepworth, A.R.; Lefevre, C.; Hartmann, P.E.; Geddes, D.T.; Hassiotou, F. Human milk microRNA and total RNA differ depending on milk fractionation. J. Cell. Biochem. 2015, 116, 2397-2407. [CrossRef] 
40. Andreas, N.J.; Kampmann, B.; Mehring Le-Doare, K. Human breast milk: A review on its composition and bioactivity. Early Hum. Dev. 2015, 91, 629-635. [CrossRef]

41. Armstrong, J.; Reilly, J.J. Breastfeeding and lowering the risk of childhood obesity. Lancet 2002, 359, $2003-2004$. [CrossRef]

42. Horta, B.L.; Loret de Mola, C.; Victora, C.G. Long-term consequences of breastfeeding on cholesterol, obesity, systolic blood pressure and type 2 diabetes: A systematic review and meta-analysis. Acta Paediatr. 2015, 104, 30-37. [CrossRef] [PubMed]

43. Yan, J.; Liu, L.; Zhu, Y.; Huang, G.; Wang, P.P. The association between breastfeeding and childhood obesity: A meta-analysis. BMC Public Health 2014, 14, 1267. [CrossRef] [PubMed]

44. Brunner, S.; Schmid, D.; Zang, K.; Much, D.; Knoeferl, B.; Kratzsch, J.; Amann-Gassner, U.; Bader, B.L.; Hauner, H. Breast milk leptin and adiponectin in relation to infant body composition up to 2 years. Pediatr. Obes. 2015, 10, 67-73. [CrossRef]

45. Palou, A.; Pico, C. Leptin intake during lactation prevents obesity and affects food intake and food preferences in later life. Appetite 2009, 52, 249-252. [CrossRef]

46. Pico, C.; Sanchez, J.; Oliver, P.; Miralles, O.; Ceresi, E.; Palou, A. Role of leptin present in maternal milk in the control of energy balance during the post-natal period. Genes Nutr. 2007, 2, 139-141. [CrossRef]

47. Andreas, N.J.; Hyde, M.J.; Gale, C.; Parkinson, J.R.; Jeffries, S.; Holmes, E.; Modi, N. Effect of maternal body mass index on hormones in breast milk: A systematic review. PLoS ONE 2014, 9, e115043. [CrossRef]

48. Schuster, S.; Hechler, C.; Gebauer, C.; Kiess, W.; Kratzsch, J. Leptin in maternal serum and breast milk: Association with infants' body weight gain in a longitudinal study over 6 months of lactation. Pediatr. Res. 2011, 70, 633-637. [CrossRef]

49. Konieczna, J.; Sanchez, J.; Palou, M.; Pico, C.; Palou, A. Blood cell transcriptomic-based early biomarkers of adverse programming effects of gestational calorie restriction and their reversibility by leptin supplementation. Sci. Rep. 2015, 5, 9088. [CrossRef]

50. Palou, M.; Pico, C.; McKay, J.A.; Sanchez, J.; Priego, T.; Mathers, J.C.; Palou, A. Protective effects of leptin during the suckling period against later obesity may be associated with changes in promoter methylation of the hypothalamic pro-opiomelanocortin gene. Br. J. Nutr. 2011, 106, 769-778. [CrossRef]

51. Pico, C.; Jilkova, Z.M.; Kus, V.; Palou, A.; Kopecky, J. Perinatal programming of body weight control by leptin: Putative roles of AMP kinase and muscle thermogenesis. Am. J. Clin. Nutr. 2011, 94, 1830S-1837S. [CrossRef] [PubMed]

52. Parsons, T.J.; Power, C.; Manor, O. Fetal and early life growth and body mass index from birth to early adulthood in 1958 British cohort: Longitudinal study. BMJ 2001, 323, 1331-1335. [CrossRef] [PubMed] 